HD-THEP-00-55

IFUP-TH/2000-41

December 2000

\title{
Evolution equations for the effective four-quark interactions in QCD
}

\author{
Enrico Meggiolaro \\ Dipartimento di Fisica, \\ Università di Pisa, \\ Via Buonarroti 2, \\ I-56127 Pisa, Italy. \\ Christof Wetterich \\ Institut für Theoretische Physik, \\ Universität Heidelberg, \\ Philosophenweg 16, \\ D-69120 Heidelberg, Germany.
}

\begin{abstract}
A nonperturbative renormalization group equation describes how the momentum dependent four-quark vertex depends on an infrared cutoff. We find a quasilocal one-particle irreducible piece generated by (anomaly-free) multi-gluon exchange. It becomes important at a cutoff scale where scalar and pseudoscalar meson-bound states are expected to play a role. This interaction remains subleading as compared to the effective one-gluon exchange contribution. The local instanton induced four-quark interaction becomes dominant at a scale around $800 \mathrm{MeV}$. In absence of a gluon mass the strong dependence of the one-gluon exchange on the transferred momentum indicates that the pointlike interactions of the Nambu-Jona-Lasinio model cannot give a very accurate description of QCD. A pointlike effective four-quark interaction becomes more realistic in case of spontaneous color symmetry breaking.
\end{abstract}




\section{Introduction}

The Nambu-Jona-Lasinio (NJL) model [1] has often been used as a simple model for lowmomentum strong interactions. After inclusion of the chiral anomaly it gives a relatively successful description of the chiral symmetry aspects of QCD and the associated properties of mesons [2]. A renormalization-group treatment based on exact flow equations has provided a consistent picture of the chiral aspects of the high temperature phase transition for two light quark flavors [3]. On the other hand it is well known that crucial properties of long-distance QCD such as confinement cannot be accounted for by this model. The NJL-model shares the chiral symmetries of QCD whereas color symmetry appears only as a global symmetry. It is based on a pointlike four-quark interaction

$$
\Gamma_{\Lambda_{\mathrm{NJL}}}^{(\mathrm{NJL})}=\int \mathrm{d}^{4} x\left\{i \bar{\psi}_{a}^{i} \gamma^{\mu} \partial_{\mu} \psi_{i}^{a}+2 \lambda_{\sigma}^{(\mathrm{NJL})}\left(\bar{\psi}_{L b}^{i} \psi_{R i}^{a}\right)\left(\bar{\psi}_{R a}^{j} \psi_{L j}^{b}\right)\right\}
$$

where $\Lambda_{\mathrm{NJL}}$ indicates that only fluctuations with momenta $q^{2}<\Lambda_{\mathrm{NJL}}^{2}$ should be included in the functional integral. (Here $i, j=1,2,3$ and $a, b=1,2,3$ are the color and flavor indices, respectively.) The quartic interaction may be supplemented by a local anomaly term which is sixth order in the quark fields for three light flavors. Also extensions with four-quark interactions in the vector channel have been investigated [2].

From the perspective of QCD, integrating out the gluon fields as well as all fermion fluctuations with (covariant) momenta larger than $\Lambda_{\mathrm{NJL}}$ should lead to an effective action containing pieces like (1.1), together with possibly quite complicated additional multifermion interactions. The question arises under which circumstances and for which problems the NJL model (1.1) can be considered as a good approximation to the multi-quark interactions obtained from QCD. As a first step towards an answer we compute in this paper the four-quark vertex with the index structure appearing in Eq. (1.1). An investigation of its momentum dependence serves as a test if the pointlike interaction of the NJL model is reasonable.

Our method to explore the physics at the nonperturbative scale $\Lambda_{\mathrm{NJL}} \approx(500-800)$ $\mathrm{MeV}$ is based on truncations of the exact nonperturbative renormalization group equation for the effective average action [4, 5, 6, 7, 8]. An earlier study [9] has followed the running of the momentum-dependent effective four-quark interaction with initial conditions "mimicking" QCD. In contrast, we start in this paper at short distances from standard perturbative QCD. We observe that a one-particle-irreducible (1PI) four-quark 
interaction is generated by box-type diagrams and becomes indeed approximately pointlike for scales of the order $\Lambda_{\mathrm{NJL}}$. Nevertheless, the contribution of the one-gluon exchange to the effective four-quark vertex retains the characteristic momentum dependence of a particle exchange in the $t$-channel. This contribution dominates quantitatively over the NJL-interaction. Another pointlike interaction arises from instanton effects. It becomes dominant at a cutoff scale around $800 \mathrm{MeV}$. We show the different contributions to the four-quark vertex in Fig. 2, where we present the case of massless gluons. For massless gluons a pointlike NJL-interaction of the type (1.1) seems not to be a realistic approximation to the effective four-quark vertex in QCD. The hypothesis that gluons acquire a mass $M_{V} \approx 850 \mathrm{MeV}$ from spontaneous color symmetry breaking [10] is discussed later (Sect. 5). For massive gluons the approximation of pointlike four-quark interactions can be defended. Nevertheless, any quantitatively reliable approximation should retain the one-gluon exchange in addition to the instanton interaction and the NJL-interaction (1.1). This is the setting used for gluon-meson duality [10.

The paper is organized as follows. In Sect. 2 we derive a truncated nonperturbative flow equation for the scalar-exchange-like effective momentum-dependent four-quark interaction in QCD. This effective one-particle irreducible interaction arises from box-type diagrams. The "box interaction" is relevant for the physics of scalar and pseudoscalar mesons and therefore for spontaneous chiral symmetry breaking. In the limit where it becomes independent of the momentum exchanged in the $t$-channel it can be described by meson exchange. In Sect. 3 we study, by numerical methods, the above-mentioned

flow equation. We also present the perturbative expansion of the flow equation and its solution and comment about some checks on the validity of our approximations. In Sect. 4 we compare the box interaction and the local instanton-induced four-quark interaction. In Sect. 5 we discuss the hypothesis of spontaneous color symmetry breaking, and a summary and conclusions are drawn in Sect. 6 .

\section{Nonperturbative flow equations}

\section{i) EFFECTIVE AVERAGE ACTION}

The effective average action $\Gamma_{k}$ [4] generalizes the generating functional for the 1PI correlation functions $\Gamma$ (effective action) for a situation where only fluctuations with (co- 
variant) momenta $q^{2}>k^{2}$ are included in the functional integral. It obtains by introducing an infrared cutoff $\sim k$ into the original functional integral such that for $k \rightarrow 0$ one recovers the effective action $\Gamma=\Gamma_{k \rightarrow 0}$. For a given $\Gamma_{k}$ at nonzero $k$ the computation of $\Gamma$ from $\Gamma_{k}$ involves only the fluctuations with $q^{2}<k^{2}$. This is the setting one needs for the effective NJL model. Our aim is therefore the computation of $\Gamma_{k}$ for $k=\Lambda_{\mathrm{NJL}}$. Since we do not know the precise value of $\Lambda_{\mathrm{NJL}}$ we will consider arbitrary $k$ in the appropriate nonperturbative range. More precisely, the NJL model is formulated in terms of quark fields. Since we start with the QCD action involving both quarks and gluons, the gluons should be integrated out. This may be done by retaining the infrared cutoff only for the fermionic fields. One then has to solve the gluon field equations obtained from $\Gamma_{k}$ as a functional of the quark fields and reinsert the solution into $\Gamma_{k}$ [11, [12]. Typically, we will not integrate out the gluon fluctuations with momenta smaller than $k$ in this paper. Our results will nevertheless be a good guide for the qualitative characteristics of the effective four-quark interactions.

The effective average action $\Gamma_{k}[A, c, \bar{c}, \psi, \bar{\psi}]$ for gluons $A$, ghosts $c$ and quarks $\psi$ obtains by a Legendre transform from a functional integral involving the QCD classical action and an infrared regulator:

$$
S_{k}[A, c, \bar{c}, \psi, \bar{\psi}]=S_{\text {gauge }}[A, c, \bar{c}]+\Delta S_{k, \text { gauge }}[A, c, \bar{c}]+S_{F}[A, \psi, \bar{\psi}]+\Delta S_{k F}[\psi, \bar{\psi}] .
$$

Here the fermionic action $S_{F}$ is given by

$$
S_{F}[A, \psi, \bar{\psi}]=\int \mathrm{d}^{4} x \bar{\psi}_{a}^{i} i \gamma^{\mu}\left(D_{\mu}[A]\right)_{i}^{j} \psi_{j}^{a}
$$

with $\left(D_{\mu}[A]\right)_{i}{ }^{j}=\delta_{i}^{j} \partial_{\mu}-i g A_{\mu}^{z}\left(T^{z}\right)_{i}{ }^{j}$ the covariant derivative in the fundamental representation. Here $a$ denotes the flavor and we use two sorts of color indices $z=1, \ldots, N_{c}^{2}-1$ and $i, j=1, \ldots, N_{c}$ for a gauge group $S U\left(N_{c}\right)$. We consider, for simplicity, $N_{f}$ massless quarks: $a=1, \ldots, N_{f}$. Throughout this paper we work in four-dimensional Euclidean space, so that the matrices $\gamma^{\mu}$ in the previous equation are the (hermitean) Euclidean $\gamma$-matrices. The gauge part $S_{\text {gauge }}$ consists of three pieces

$$
S_{\text {gauge }}[A, c, \bar{c}]=S_{Y M}[A]+S_{g f}[A]+S_{g h}[A, c, \bar{c}]
$$

where $S_{Y M}$ is the Yang-Mills action, given by:

$$
S_{Y M}[A]=\frac{1}{4} \int \mathrm{d}^{4} x F_{\mu \nu}^{z} F_{\mu \nu}^{z}
$$


with

$$
F_{\mu \nu}^{z}=\partial_{\mu} A_{\nu}^{z}-\partial_{\nu} A_{\mu}^{z}+g f^{z y w} A_{\mu}^{y} A_{\nu}^{w}
$$

The gauge-fixing part $S_{g f}$ reads

$$
S_{g f}[A]=\frac{1}{2 \alpha} \int \mathrm{d}^{4} x \partial_{\mu} A_{\mu}^{z} \partial_{\nu} A_{\nu}^{z}
$$

where $\alpha$ is the gauge parameter, and the ghost action $S_{g h}$ is given by

$$
S_{g h}[A, c, \bar{c}]=\int \mathrm{d}^{4} x \partial_{\mu} \bar{c}^{z}\left(\delta^{z w} \partial_{\mu}+g f^{z y w} A_{\mu}^{y}\right) c^{w}
$$

The infrared cutoff modifies the propagators. For the fermions, the explicit expression $\Delta S_{k F}[\psi, \bar{\psi}]$ reads in momentum space 9,12

$$
\Delta S_{k F}[\psi, \bar{\psi}]=\int \frac{\mathrm{d}^{4} q}{(2 \pi)^{4}} \bar{\psi}_{a}^{i}(q) R_{k F}(q) \psi_{i}^{a}(q)
$$

where we choose here

$$
\begin{aligned}
R_{k F}(q) & =\gamma^{\mu} q_{\mu} r_{k F}\left(q^{2}\right) \\
r_{k F}\left(q^{2}\right) & =\frac{\mathrm{e}^{-q^{2} / k^{2}}}{1-\mathrm{e}^{-q^{2} / k^{2}}}
\end{aligned}
$$

In particular, the (regularized) free fermion propagator turns out to be:

$$
\left[G_{2, k}^{(F)}(q)\right]_{i j}^{a b}=\delta^{a b} \delta_{i j} \frac{\gamma^{\mu} q_{\mu}}{q^{2}\left[1+r_{k F}\left(q^{2}\right)\right]}=\delta^{a b} \delta_{i j} \gamma^{\mu} q_{\mu} \frac{1-\mathrm{e}^{-q^{2} / k^{2}}}{q^{2}}
$$

The gauge infrared cutoff term $\Delta S_{k, \text { gauge }}[A, c, \bar{c}]$ is the sum of a gluon infrared cutoff term and a ghost infrared cutoff term: $\Delta S_{k, \text { gauge }}[A, c, \bar{c}]=\Delta S_{k A}[A]+\Delta S_{k, g h}[c, \bar{c}]$. The explicit expression of the gluon infrared cutoff term $\Delta S_{k A}[A]$ is given by [6, 8]

$$
\Delta S_{k A}[A]=\frac{1}{2} \int \frac{\mathrm{d}^{4} p}{(2 \pi)^{4}} A_{\mu}^{z}(-p) R_{\mu \nu}^{k A}(p) A_{\nu}^{z}(p)
$$

where

$$
R_{\mu \nu}^{k A}(p)=\left[\delta_{\mu \nu}+\left(\frac{1}{\alpha}-1\right) \frac{p_{\mu} p_{\nu}}{p^{2}}\right] R_{k A}\left(p^{2}\right)
$$


with

$$
R_{k A}\left(p^{2}\right)=p^{2} \frac{\mathrm{e}^{-p^{2} / k^{2}}}{1-\mathrm{e}^{-p^{2} / k^{2}}} .
$$

Finally, the ghost infrared cutoff term $\Delta S_{k, g h}[c, \bar{c}]$ reads

$$
\Delta S_{k, g h}[c, \bar{c}]=\int \frac{\mathrm{d}^{4} p}{(2 \pi)^{4}} \bar{c}^{z}(-p) R_{k, g h}\left(p^{2}\right) c^{z}(p)
$$

where

$$
R_{k, g h}\left(p^{2}\right)=p^{2} \frac{\mathrm{e}^{-p^{2} / k^{2}}}{1-\mathrm{e}^{-p^{2} / k^{2}}} .
$$

With this choice, the regularized ghost propagator becomes

$$
\left[G_{2, k}^{(g h)}(q)\right]^{z y}=\frac{\delta^{z y}}{p^{2}+R_{k, g h}\left(p^{2}\right)}=\delta^{z y} \frac{1-\mathrm{e}^{-p^{2} / k^{2}}}{p^{2}}
$$

It is well known [6, 7, 8] that the presence of the infrared cutoff explicitly breaks gauge and hence BRS invariance: in particular, a gluon mass term appears. A background field identity [6] or modified Slavnov-Taylor identities [8, 13] can be derived, which are valid for $k>0$. They guarantee the BRS invariance of the theory for $k \rightarrow 0$. Within a perturbative expansion of $\Gamma_{k}$, one can derive an equation for a $k$-dependent mass term for the gauge fields implied by the modified Slavnov-Taylor identities. For pure Yang-Mills theories (i.e., with no fermions), regularized with the above infrared cutoff term (2.11)-(2.13), one finds the following result, at the leading perturbative order [8, 11]:

$$
m_{k A}^{2}=\frac{3 N_{c} g_{k}^{2}}{128 \pi^{2}} k^{2}(\alpha-1)
$$

Here $\alpha$ is the gauge parameter, introduced in Eq. (2.6), and $g_{k}$ is the renormalized ( $k$-dependent) coupling constant. In the theory with regularized fermions that we are considering, a contribution to the gluon mass also appears from the fermion part of the gluon self-energy. One finds, at the leading perturbative order:

$$
m_{k F}^{2}=\frac{3 N_{f} g_{k}^{2}}{32 \pi^{2}} k^{2}
$$


When including the gluon mass, the regularized gluon propagator becomes, in the Feynman gauge $\alpha=1$ :

$$
\left[G_{2, k}^{(A)}(p)\right]_{\mu \nu}^{z y}=\frac{\delta_{\mu \nu} \delta^{z y}}{p^{2}+m_{k}^{2}+R_{k A}\left(p^{2}\right)}
$$

where

$$
m_{k}^{2}=m_{k A}^{2}+m_{k F}^{2} .
$$

(Indeed, $m_{k A}^{2}=0$ at the perturbative order $O\left(g_{k}^{2}\right)$ in the Feynman gauge.) In other words, using the expression (2.13) for $R_{k A}\left(p^{2}\right)$ :

$$
\left[G_{2, k}^{(A)}(p)\right]_{\mu \nu}^{z y}=\delta_{\mu \nu} \delta^{z y} \frac{1-\mathrm{e}^{-p^{2} / k^{2}}}{p^{2}+m_{k}^{2}\left(1-\mathrm{e}^{-p^{2} / k^{2}}\right)} .
$$

We shall concentrate on the part $\Gamma_{k}^{(F)}[\psi, \bar{\psi}]$ of the effective average action which depends only on the fermion fields:

$$
\Gamma_{k}[A, c, \bar{c}, \psi, \bar{\psi}]=\Gamma_{k}^{(F)}[\psi, \bar{\psi}]+\Gamma_{k}^{(A)}[A, c, \bar{c}, \psi, \bar{\psi}]
$$

In general, it can be expanded [11, [12] as

$$
\Gamma_{k}^{(F)}[\psi, \bar{\psi}]=\Gamma_{2, k}^{(F)}[\psi, \bar{\psi}]+\Gamma_{4, k}^{(F)}[\psi, \bar{\psi}]+\ldots
$$

where $\Gamma_{2 n, k}^{(F)}[\psi, \bar{\psi}]$ contains terms with $2 n$ fermion fields, i.e., of the form $\sim(\bar{\psi} \psi)^{n}$. For $n=1$, we have the bilinear term

$$
\Gamma_{2, k}^{(F)}[\psi, \bar{\psi}]=\int \frac{\mathrm{d}^{4} q}{(2 \pi)^{4}} \bar{\psi}_{a}^{i}(q) \gamma^{\mu} q_{\mu} \psi_{i}^{a}(q),
$$

Similar to Refs. [9, 12], we truncate the effective action by omitting $\Gamma_{2 n, k}^{(F)}[\psi, \bar{\psi}]$ with $n \geq 3$ and we concentrate on the behavior of the four-quark interaction $(n=2)$. For $\Gamma_{4, k}^{(F)}[\psi, \bar{\psi}]$ we adopt a chirally-invariant parametrization [12]:

$$
\begin{aligned}
\Gamma_{4, k}^{(F)} & {[\psi, \bar{\psi}]=-\int \frac{\mathrm{d}^{4} p_{1}}{(2 \pi)^{4}} \int \frac{\mathrm{d}^{4} p_{2}}{(2 \pi)^{4}} \int \frac{\mathrm{d}^{4} p_{3}}{(2 \pi)^{4}} \int \frac{\mathrm{d}^{4} p_{4}}{(2 \pi)^{4}}(2 \pi)^{4} \delta^{(4)}\left(p_{1}+p_{2}-p_{3}-p_{4}\right) } \\
& \times\left\{\lambda_{\sigma}\left(p_{1}, p_{2}, p_{3}, p_{4}\right) \mathcal{M}_{\sigma}\left(p_{1}, p_{2}, p_{3}, p_{4}\right)+\lambda_{\rho}\left(p_{1}, p_{2}, p_{3}, p_{4}\right) \mathcal{M}_{\rho}\left(p_{1}, p_{2}, p_{3}, p_{4}\right)\right. \\
& \left.+\lambda_{p}\left(p_{1}, p_{2}, p_{3}, p_{4}\right) \mathcal{M}_{p}\left(p_{1}, p_{2}, p_{3}, p_{4}\right)+\ldots\right\}
\end{aligned}
$$

*We omit in this paper the running of the fermion wave function renormalization. 
where the four-fermion couplings $\lambda_{i}$ have the property:

$$
\lambda_{i}\left(p_{1}, p_{2}, p_{3}, p_{4}\right)=\lambda_{i}\left(-p_{4},-p_{3},-p_{2},-p_{1}\right)
$$

and the usual invariant quantities $s$ and $t$ read

$$
s=\left(p_{1}+p_{2}\right)^{2}=\left(p_{3}+p_{4}\right)^{2}, \quad t=\left(p_{1}-p_{3}\right)^{2}=\left(p_{2}-p_{4}\right)^{2} .
$$

The four-fermion operators $\mathcal{M}_{i}$ are defined as:

$$
\begin{aligned}
\mathcal{M}_{\sigma}\left(p_{1}, p_{2}, p_{3}, p_{4}\right)= & -\frac{1}{2}\left[\bar{\psi}_{a}^{i}\left(-p_{1}\right) \psi_{i}^{b}\left(p_{2}\right)\right]\left[\bar{\psi}_{b}^{j}\left(p_{4}\right) \psi_{j}^{a}\left(-p_{3}\right)\right] \\
& +\frac{1}{2}\left[\bar{\psi}_{a}^{i}\left(-p_{1}\right) \gamma^{5} \psi_{i}^{b}\left(p_{2}\right)\right]\left[\bar{\psi}_{b}^{j}\left(p_{4}\right) \gamma^{5} \psi_{j}^{a}\left(-p_{3}\right)\right] ; \\
\mathcal{M}_{\rho}\left(p_{1}, p_{2}, p_{3}, p_{4}\right)= & +\frac{1}{4}\left[\bar{\psi}_{a}^{i}\left(-p_{1}\right) \gamma^{\mu} \psi_{i}^{b}\left(p_{2}\right)\right]\left[\bar{\psi}_{b}^{j}\left(p_{4}\right) \gamma_{\mu} \psi_{j}^{a}\left(-p_{3}\right)\right] \\
& +\frac{1}{4}\left[\bar{\psi}_{a}^{i}\left(-p_{1}\right) \gamma^{\mu} \gamma^{5} \psi_{i}^{b}\left(p_{2}\right)\right]\left[\bar{\psi}_{b}^{j}\left(p_{4}\right) \gamma_{\mu} \gamma^{5} \psi_{j}^{a}\left(-p_{3}\right)\right] ; \\
\mathcal{M}_{p}\left(p_{1}, p_{2}, p_{3}, p_{4}\right)= & -\frac{1}{2 N_{c}}\left[\bar{\psi}_{a}^{i}\left(-p_{1}\right) \gamma^{\mu} \psi_{i}^{a}\left(-p_{3}\right)\right]\left[\bar{\psi}_{b}^{j}\left(p_{4}\right) \gamma_{\mu} \psi_{j}^{b}\left(p_{2}\right)\right] .
\end{aligned}
$$

If $\lambda_{\sigma}$ does not depend on $t$, the term $\mathcal{M}_{\sigma}$ describes the exchange of color-singlet, flavornonsinglet spin-0 scalar quark-antiquark states in the $s$ channel: they correspond to the spin-0 pseudoscalar and scalar mesons. Similarly, $\mathcal{M}_{\rho}$ describes the exchange of colorsinglet, flavor-nonsinglet spin-1 vector states in the $s$ channel: they correspond to the spin-1 vector and axial vector mesons. Finally, $\mathcal{M}_{p}$ describes the propagation of a colorand flavor-singlet spin-1 vector state in the $t$ channel: these are the quantum numbers of the pomeron. We concentrate on the analysis (both analytical and numerical) of the evolution equation for the scalar-like effective four-fermion coupling $\lambda_{\sigma}$, which multiplies $\mathcal{M}_{\sigma}$ and is relevant for the physics of scalar and pseudoscalar mesons and therefore for spontaneous chiral symmetry breaking. The dots in Eq. (2.25) stand for more complicated momentum structures, the contribution of the chiral anomaly (to be discussed in Sect. 4) and terms breaking the chiral flavor symmetry.

\section{ii) FLOW EQUATIONS}

The exact flow equation for $\Gamma_{k}$ can be written as a formal derivative of a renormalization group improved one-loop expression [4]

$$
\partial_{k} \Gamma_{k}=\frac{1}{2} \operatorname{STr}\left[\tilde{\partial}_{k} \ln \left(\Gamma_{k}^{(2)}+R_{k}\right)\right]
$$


where $\Gamma_{k}^{(2)}$ is the second functional derivative with respect to the fields and $\tilde{\partial}_{k}$ acts only on $R_{k}$ and not on $\Gamma_{k}^{(2)}$, i.e.:

$$
\tilde{\partial}_{k} \equiv \frac{\partial R_{k}}{\partial k} \frac{\partial}{\partial R_{k}} .
$$

Here $R_{k}$ is to be interpreted as a matrix $R_{k}=\operatorname{diag}\left(R_{k A}, R_{k, g h}, R_{k F}\right)$. The trace involves a summation over indices and a momentum integration and "STr" indicates an additional minus sign for the fermionic part. Flow equations for $n$-point functions follow from appropriate functional derivatives of Eq. (2.29) with respect to the fields. For their derivation it is sufficient to evaluate the corresponding one-loop expressions in presence of the infrared cutoff (with the vertices and propagators derived from $\Gamma_{k}$ ) and then to take a formal $\tilde{\partial}_{k}$ derivative. This permits the use of (one-loop) Feynman diagrams and standard perturbative techniques.

Using these techniques, we have derived the flow equation for the four-fermion (1PI) coupling $\lambda_{\sigma}$ by computing the relevant one-loop diagrams from the effective average action $\Gamma_{k}[A, c, \bar{c}, \psi, \bar{\psi}]$ : these diagrams are shown in Fig. 1. Our truncation includes only two vertices: i) the usual quark-gluon vertex and ii) the four-fermion (1PI) vertex $\lambda_{\sigma} \mathcal{M}_{\sigma}$. We have extracted from the relevant diagrams the contributions to the $\sigma$-type four-fermion vertex $\mathcal{M}_{\sigma}$ and then performed a formal $\tilde{\partial}_{k}$ derivative in the sense of Eq. (2.30). In this picture (which is different from the one used in Ref. [9]) the gluons are not integrated out. Therefore, in addition to the contributions to the flow equation coming from the infrared cutoff in the fermion lines inside a given Feynman diagram, one has to consider also the ones from the infrared cutoff in the gluon lines.

iii) TRUNCATIONS AND THE FLOW EQUATION FOR $\lambda_{\sigma}$

In general, the function $\lambda_{\sigma}$ can depend on six independent Lorentz-invariant products of the momenta $\lambda_{\sigma}=\lambda_{\sigma}\left(s, t, p_{1}^{2}, p_{2}^{2}, p_{3}^{2}, p_{4}^{2}\right)$. We have derived the evolution equation of $\lambda_{\sigma}$ for the particular configuration of momenta $\left(p_{1}, p_{2}, p_{3}, p_{4}\right)=(p,-p,-p, p)$, so that $s=0, t=4 p^{2}$. In order to simplify the momentum structure, we have made a further truncation, namely that $\lambda_{\sigma}$ depends only on the invariant $t: \lambda_{\sigma} \simeq \lambda_{\sigma}(t)$. Furthermore, our truncation restricts the quark-gluon interactions to minimal coupling with a coupling constant depending on the gluon momentum. We use the classical form of the (regularized) gluon propagatori. Our truncation omits the influence of all multiquark interactions except those proportional $\mathcal{M}_{\sigma}(2.28)$. Moreover, we neglect the possible effects from a $q^{2}-$

\footnotetext{
${ }^{\dagger}$ See [13, 14] for an investigation of the $q^{2}$-dependence of the gluon propagator by means of nonperturbative flow equations.
} 
and $k$-dependent fermion wave-function renormalization $Z_{\psi, k}$.

With these approximations, we find the following result:

$$
\begin{aligned}
& k \partial_{k} \lambda_{\sigma}(t)= \\
& \quad=-\frac{16 N_{c}}{k^{2}} \int \frac{\mathrm{d}^{4} q}{(2 \pi)^{4}}\left\{4 \pi^{2}\left(1-\frac{3}{2 N_{c}^{2}}\right) \alpha_{k}\left((p-q)^{2}\right) \alpha_{k}\left((p+q)^{2}\right)\right. \\
& \quad \times q^{2} F_{k}(q) G_{k}(p-q)\left[\mathrm{e}^{-q^{2} / k^{2}} G_{k}(p+q)+F_{k}(q) \Delta_{k}(p+q)\right] \\
& \quad-\pi \alpha_{k}\left((p+q)^{2}\right) q^{2}\left(\frac { 1 } { 2 N _ { c } ^ { 2 } } \lambda _ { \sigma } ( 4 p ^ { 2 } ) \left[\mathrm{e}^{-q^{2} / k^{2}} F_{k}(2 p+q) G_{k}(p+q)\right.\right. \\
& \left.\quad+\mathrm{e}^{-(2 p+q)^{2} / k^{2}} F_{k}(q) G_{k}(p+q)+F_{k}(q) F_{k}(2 p+q) \Delta_{k}(p+q)\right] \\
& \left.\quad-\left(2-\frac{3}{2 N_{c}^{2}}\right) \lambda_{\sigma}\left((p-q)^{2}\right) F_{k}(q)\left[2 \mathrm{e}^{-q^{2} / k^{2}} G_{k}(p+q)+F_{k}(q) \Delta_{k}(p+q)\right]\right) \\
& \left.\quad+\lambda_{\sigma}\left((p-q)^{2}\right) \lambda_{\sigma}\left((p+q)^{2}\right) q^{2} \mathrm{e}^{-q^{2} / k^{2}} F_{k}(q)\right\} .
\end{aligned}
$$

Here we use the following shorthands:

$$
\begin{aligned}
& F_{k}(q)=\frac{1}{q^{2}\left[1+r_{k F}\left(q^{2}\right)\right]}=\frac{1-\mathrm{e}^{-q^{2} / k^{2}}}{q^{2}} ; \\
& G_{k}(q)=\frac{1}{q^{2}+m_{k}^{2}+R_{k A}\left(q^{2}\right)}=\frac{1-\mathrm{e}^{-q^{2} / k^{2}}}{q^{2}+m_{k}^{2}\left(1-\mathrm{e}^{-q^{2} / k^{2}}\right)} ; \\
& \Delta_{k}(q)=\frac{\left(q^{2}\right)^{2} \mathrm{e}^{-q^{2} / k^{2}}}{\left[q^{2}+m_{k}^{2}\left(1-\mathrm{e}^{-q^{2} / k^{2}}\right)\right]^{2}},
\end{aligned}
$$

and employ

$$
\begin{aligned}
& \widetilde{\partial}_{k} F_{k}(q)=\frac{\partial r_{k F}\left(q^{2}\right)}{\partial k} \frac{\partial F_{k}(q)}{\partial r_{k F}\left(q^{2}\right)}=-\frac{2}{k^{3}} \mathrm{e}^{-q^{2} / k^{2}}, \\
& \widetilde{\partial}_{k} G_{k}(q)=\frac{\partial R_{k A}\left(q^{2}\right)}{\partial k} \frac{\partial G_{k}(q)}{\partial R_{k A}\left(q^{2}\right)}=-\frac{2}{k^{3}} \Delta_{k}(q) .
\end{aligned}
$$

The first two terms in the r.h.s. of Eq. (2.31) (i.e., those proportional to $\sim \alpha_{k}^{2}$ or $\sim \alpha_{k} \lambda_{\sigma}$ ) reflect the exchange of gauge bosons [see Fig. 1a) $\div 1$ c)]. The last term (i.e., the one proportional to $\left.\sim \lambda_{\sigma}^{2}\right)$ corresponds to the result of Ref. [9] for the truncation $\lambda_{\sigma}=\lambda_{\sigma}(t)$ [see Fig. 1d)]. 


\section{iv) RG-IMPROVEMENT OF THE GAUGE COUPLING}

The flow equation (2.31) is "RG-improved" in the sense that we use everywhere a "running" coupling constant $\alpha_{k}\left(q^{2}\right)=g_{k}^{2}\left(q^{2}\right) / 4 \pi$. Instead of computing $\alpha_{k}\left(q^{2}\right)$ by its own flow equation, we use here an "ad hoc" relation to the two-loop running gauge coupling:

$$
\alpha(\mu)=\frac{1}{4 \pi \beta_{0} \ln \left(\mu^{2} / \Lambda_{Q C D}^{2}\right)}\left[1-\frac{\beta_{1}}{\beta_{0}^{2}} \frac{\ln \left[\ln \left(\mu^{2} / \Lambda_{Q C D}^{2}\right)\right]}{\ln \left(\mu^{2} / \Lambda_{Q C D}^{2}\right)}\right] .
$$

Here $\beta_{0}$ and $\beta_{1}$ are the two first coefficients of the QCD $\beta$-function

$$
\beta(g)=\mu \frac{d g}{d \mu}=-\beta_{0} g^{3}-\beta_{1} g^{5}+\ldots
$$

which, for a gauge group $S U\left(N_{c}\right)$ and $N_{f}$ quark flavors, are given by:

$$
\beta_{0}=\frac{1}{(4 \pi)^{2}}\left[\frac{11 N_{c}-2 N_{f}}{3}\right], \quad \beta_{1}=\frac{1}{(4 \pi)^{4}}\left[\frac{34}{3} N_{c}^{2}-\left(\frac{13 N_{c}^{2}-3}{3 N_{c}}\right) N_{f}\right]
$$

such that, for $N_{c}=3$ and $N_{f}=3$ :

$$
\alpha(\mu)=\frac{4 \pi}{9 \ln \left(\mu^{2} / \Lambda_{Q C D}^{2}\right)}\left[1-\frac{64}{81} \frac{\ln \left[\ln \left(\mu^{2} / \Lambda_{Q C D}^{2}\right)\right]}{\ln \left(\mu^{2} / \Lambda_{Q C D}^{2}\right)}\right] .
$$

For the relation between $q^{2}, k^{2}$ and $\mu^{2}$, we use two different ansätze: (A) $\mu^{2}=q^{2}+k^{2}$, and (B) $\mu^{2}=k^{2}$. The first one (A) is motivated by the fact that both $q^{2}$ and $k^{2}$ act as effective infrared cutoffs such that essentially the larger one counts. The second one (B) neglects the dependence of the vertex on the gluon momentum. The overall mass scale needs in addition the unknown ratio between $\Lambda_{Q C D}$ in the scheme relevant for the flow equations (ERGE-scheme) to the $\overline{\mathrm{MS}}$ scheme. We therefore report all quantities with dimension of $[\text { mass }]^{n}$ in units of $\left(\mathrm{GeV} \cdot \Lambda_{Q C D} / \Lambda \frac{(3)}{\mathrm{MS}}\right)^{n}$, where $\Lambda \frac{(3)}{\mathrm{MS}}$ is the fundamental mass scale of QCD in the $\overline{\mathrm{MS}}$ renormalization scheme (two-loop), with $N_{f}=3$. The experimental value $\alpha_{s}\left(M_{Z}\right)=0.118(3)$ corresponds to $\Lambda \frac{(3)}{\mathrm{MS}} \simeq 360 \mathrm{MeV}$. For comparison we will also use a fixed value of $\alpha$ corresponding to the scale $\mu=k_{c}=1.5 \cdot \Lambda_{Q C D} / \Lambda \frac{(3)}{\mathrm{MS}} \mathrm{GeV}$, i.e., $\alpha_{k_{c}} \simeq 0.34$ [ansatz $(\mathrm{C})]$. 


\section{v) ONE-GLUON-EXCHANGE CONTRIBUTION}

The term $\lambda_{\sigma} \mathcal{M}_{\sigma}$ describes only the contribution to the effective $\sigma$-type vertex coming from the 1PI graphs. A four-quark interaction with a similar structure arises also from one-gluon exchange which is not one-particle irreducible and therefore does not contributef to $\lambda_{\sigma}$ in the present formulation. Indeed, the four-fermion vertex induced by the one-gluon exchange is given by [12]

$$
\begin{aligned}
& \Delta \Gamma^{(1 g)}=-\int \frac{\mathrm{d}^{4} p_{1}}{(2 \pi)^{4}} \int \frac{\mathrm{d}^{4} p_{2}}{(2 \pi)^{4}} \int \frac{\mathrm{d}^{4} p_{3}}{(2 \pi)^{4}} \int \frac{\mathrm{d}^{4} p_{4}}{(2 \pi)^{4}}(2 \pi)^{4} \delta^{(4)}\left(p_{1}+p_{2}-p_{3}-p_{4}\right) \times \\
& \times 2 \pi \alpha_{k}\left(\left(p_{1}-p_{3}\right)^{2}\right) \cdot G_{k}\left(p_{1}-p_{3}\right) \cdot \mathcal{M}\left(p_{1}, p_{2}, p_{3}, p_{4}\right)
\end{aligned}
$$

where

$$
\mathcal{M}\left(p_{1}, p_{2}, p_{3}, p_{4}\right)=\left[\bar{\psi}_{a}^{i}\left(-p_{1}\right) \gamma^{\mu}\left(T^{z}\right)_{i}^{j} \psi_{j}^{a}\left(-p_{3}\right)\right]\left[\bar{\psi}_{b}^{k}\left(p_{4}\right) \gamma_{\mu}\left(T_{z}\right)_{k}^{l} \psi_{l}^{b}\left(p_{2}\right)\right]
$$

By an appropriate Fierz transformation, one can show that

$$
\mathcal{M}=\mathcal{M}_{\sigma}+\mathcal{M}_{\rho}+\mathcal{M}_{p}
$$

where $\mathcal{M}_{\sigma}, \mathcal{M}_{\rho}$ and $\mathcal{M}_{p}$ have been defined in Eq. (2.28). The total contribution in the $\sigma$-channel is therefore $\sim\left(\lambda_{\sigma}+\lambda_{\sigma}^{(1 g)}\right) \mathcal{M}_{\sigma}$ with

$$
\lambda_{\sigma}^{(1 g)}(t)=2 \pi \alpha_{k}(t) \frac{1-e^{-t / k^{2}}}{t+m_{k}^{2}\left(1-e^{-t / k^{2}}\right)}
$$

This contribution remains nonlocal for $k \rightarrow 0$ if $m_{k}^{2}$ tends to zero in this limit. The size of the 1PI contribution $\lambda_{\sigma}$ should be compared with the effective coupling (2.41).

\footnotetext{
${ }^{\ddagger}$ In the formulation [9, 11, 12] the one-gluon exchange part is included in $\lambda_{\sigma}$.
} 


\section{Scale dependence of the four-quark interaction}

\section{i) PERTURBATION THEORY}

In perturbation theory the 1PI four-quark interactions arise from "box" and "cross" two-gluon exchange diagrams [see Fig. 1a) and 1b)]. For the part with the structure $\mathcal{M}_{\sigma}$ we find the perturbative value $\left(t=4 p^{2}\right)$

$$
\begin{aligned}
\lambda_{\sigma}^{(p)}(t)=16 \pi^{2} N_{c}\left(1-\frac{3}{2 N_{c}^{2}}\right) & \int \frac{\mathrm{d}^{4} q}{(2 \pi)^{4}} \alpha_{k}\left((p-q)^{2}\right) \alpha_{k}\left((p+q)^{2}\right) \\
& \times q^{2}\left[F_{k}(q)\right]^{2} G_{k}(p-q) G_{k}(p+q) .
\end{aligned}
$$

In particular, for $t=0$ and momentum-independent $\alpha_{k}[\operatorname{ansatz}(\mathrm{B})]$, the momentum integration can be performed explicitly. One finds, for $N_{c}=3$ and in the limit of a vanishing gluon mass term $m_{k}^{2}=0$ :

$$
\lambda_{\sigma}^{(p)}(t=0)=\frac{5 \alpha_{k}^{2}}{k^{2}} \cdot K_{\sigma}
$$

Here the constant

$$
\begin{aligned}
K_{\sigma} & =8 \pi^{2} k^{2} \int \frac{\mathrm{d}^{4} q}{(2 \pi)^{4}} q^{2}\left[F_{k}(q)\right]^{2}\left[G_{k}^{0}(q)\right]^{2} \\
G_{k}^{0}(q) & =\left.G_{k}(q)\right|_{m_{k}^{2}=0}=\frac{1}{q^{2}+R_{k A}\left(q^{2}\right)}=\frac{1-e^{-q^{2} / k^{2}}}{q^{2}}
\end{aligned}
$$

depends on the precise form? of the infrared cutoff. For our choice [see Eq. (2.13)] one finds

$$
K_{\sigma}=2 \cdot[5 \ln 2-3 \ln 3] \simeq 0.34
$$

For the purpose of comparison with our results in the following figures, we quote the value corresponding to $k=k_{c}=1.5 \cdot \Lambda_{Q C D} / \Lambda_{\overline{\mathrm{MS}}}^{(3)} \mathrm{GeV}\left(\alpha_{k_{c}} \simeq 0.34\right)$ :

$$
\left.\lambda_{\sigma}^{(p)}(t=0)\right|_{k=k_{c}} \simeq 8.7 \times 10^{-2}\left(\mathrm{GeV} \cdot \Lambda_{Q C D} / \Lambda_{\overline{\mathrm{MS}}}^{(3)}\right)^{-2} .
$$

In order to get a feeling for the relative strength of the $\sigma$-channel as compared to other types of 1PI four-quark interactions, we also present here the full one-loop 1PI interaction

${ }^{*}$ For the fermion cutoff used in [5] one has to replace $F^{2} \rightarrow F / q^{2}$. 
for zero external momenta (i.e., $s=0, t=0$ ). For an arbitrary gauge parameter $\alpha$ one obtains the effective (local) four-quark interaction

$$
\begin{aligned}
\mathcal{L}_{4, k}^{(F)(p)=} & -\frac{g_{k}^{4}}{64 \pi^{2} k^{2}} K_{\sigma} \\
& \left\{\left(\frac{9}{4}+\frac{9}{2} \alpha+\frac{3}{4} \alpha^{2}\right)\left[\left(\bar{\psi}_{a}^{i} \gamma^{\mu} \psi_{j}^{a}\right)\left(\bar{\psi}_{b}^{j} \gamma_{\mu} \psi_{i}^{b}\right)-\frac{1}{3}\left(\bar{\psi}_{a}^{i} \gamma^{\mu} \psi_{i}^{a}\right)\left(\bar{\psi}_{b}^{j} \gamma_{\mu} \psi_{j}^{b}\right)\right]\right. \\
& \left.-\frac{5}{2}\left(\bar{\psi}_{a}^{i} \gamma^{\mu} \gamma^{5} \psi_{j}^{a}\right)\left(\bar{\psi}_{b}^{j} \gamma_{\mu} \gamma^{5} \psi_{i}^{b}\right)-\frac{11}{6}\left(\bar{\psi}_{a}^{i} \gamma^{\mu} \gamma^{5} \psi_{i}^{a}\right)\left(\bar{\psi}_{b}^{j} \gamma_{\mu} \gamma^{5} \psi_{j}^{b}\right)\right\} .
\end{aligned}
$$

We note that the first term has precisely the structure of the one-gluon-exchange diagram $\sim \mathcal{M}(2.39)$. We also emphasize that there are several ways how to Fierz-transform the expression (3.6). Our truncation of keeping only $\lambda_{\sigma}$ corresponds more precisely to state which invariants are neglected.

It is straightforward to verify that the first term in the flow equation (2.31) for $\lambda_{\sigma}$ precisely corresponds to the formal $\tilde{\partial}_{k}$ derivative of the perturbative value (3.1). For $t=0$ the contribution to $k \partial_{k} \lambda_{\sigma}$ obtains by replacing in the r.h.s. of Eq. (3.2) the constant $K_{\sigma}$ by $-2 K_{\sigma} \simeq-0.68$.

\section{ii) INITIAL CONDITION FOR THE FLOW EQUATION}

In order to solve explicitly the flow equation (2.31), we need to fix the initial condition, i.e., the value of $\lambda_{\sigma}(t)$ at a certain "initial" value $k=k_{c}$. We assume that at the scale $k=k_{c}=1.5 \cdot \Lambda_{Q C D} / \Lambda_{\mathrm{MS}}^{(3)} \mathrm{GeV}$, where perturbation theory is assumed to work well, the value of $\left.\lambda_{\sigma}(t)\right|_{k=k_{c}}$ is well approximated by the leading perturbative contribution. In other words, we use as initial condition for the flow equation (2.31):

$$
\left.\lambda_{\sigma}(t)\right|_{k=k_{c}}=\left.\lambda_{\sigma}^{(p)}(t)\right|_{k=k_{c}} .
$$

We have then solved numerically the flow equation (2.31) with the initial condition (3.1), (3.7).

\section{iii) RESULTS}

In Fig. 2 we plot the resulting $\lambda_{\sigma}(t)$ (for $N_{c}=3$ and $N_{f}=3$ ) as a function of $k$, for the particular case $t=0$. The continuous line corresponds to a running gauge coupling according to the ansatz (A). The dotted line in the same figure represents $\lambda_{\sigma}(t=0)$ as a function of $k$ with the initial condition $\left.\lambda_{\sigma}(t)\right|_{k=k_{c}}=0$, instead of (3.1), (3.7). We observe the insensitivity with respect to the precise choice of the initial condition. The dot-dashed 
line is the value of $\lambda_{\sigma}(t=0)$ as a function of $k$ with the initial condition (3.1), (3.7), but using everywhere (both in the flow equation and in the initial condition) the momentumindependent coupling constant $\alpha_{k}$ defined by Eq. (2.37) with $\mu^{2}=k^{2}[$ ansatz (B)]. We observe a strong increase of $\lambda_{\sigma}$ at a scale $k \approx 400-600 \mathrm{MeV}$.

Comparison with perturbation theory is made in Fig. 3. We plot the value of $\lambda_{\sigma}^{(p)}(t=$ 0 ), defined by Eq. (3.1), as a function of $k$ (dotted line). The dashed line represents the value of $\lambda_{\sigma}^{(p)}(t=0)$, defined by Eq. (3.1), as a function of $k$, but using the momentumindependent coupling constant $\alpha_{k}$ defined by Eq. (2.37) with $\mu^{2}=k^{2}[\operatorname{ansatz}(\mathrm{B})]$. In this case, the integration in Eq. (3.1) can be performed explicitly (neglecting also the gluon mass $m_{k}$ appearing in the function $G_{k}$ ) and one obtains (for $N_{c}=3$ ) the result (3.2)-(3.4). Finally, the dot-dashed line is obtained by using this expression for $\lambda_{\sigma}^{(p)}(t=0)$, but using the fixed coupling constant $\alpha_{k}=\alpha_{k=k_{c}} \simeq 0.34[\operatorname{ansatz}(\mathrm{C})]$.

In Fig. 4 we plot $\lambda_{\sigma}(t)$ (continuous line) as a function of $t$ at the fixed value $k=0.42$. $\Lambda_{Q C D} / \Lambda \frac{(3)}{\mathrm{MS}} \mathrm{GeV}$. At this scale the approximation of the box-type four-quark interaction by a local NJL-vertex (1.1) is reasonable.

\section{iv) CHECKING THE APPROXIMATIONS}

When inserting the effective action (2.22), (2.25) into the flow equation (2.29) and extracting only the contributions to the $\sigma$-type four-fermion vertex $\mathcal{M}_{\sigma}$, one finds that in the flow equation (2.31) for the effective coupling $\lambda_{\sigma}$ also other pieces proportional to $\sim \lambda_{i} \lambda_{j}$ appear: these additional pieces are generated by the four-fermion structures $\mathcal{M}_{\rho}$ and $\mathcal{M}_{p}$ and they are put to zero in our truncation. Since only the retained contribution $\sim \lambda_{\sigma}^{2}$ is proportional to the color factor $N_{c}$, while all other terms of the form $\sim \lambda_{i} \lambda_{j}$ are suppressed, our truncated evolution equation becomes exact in the leading order in a $1 / N_{c}$ expansion. Moreover, we have checked that a multiplication of the term proportional to $\lambda_{\sigma}^{2}$ in the flow equation (2.31) by a factor of ten does not strongly influence the results. The solution $\lambda_{\sigma}(t)$ of the modified flow equation is very near to the real solution of Eq. (2.31) reported in Figs. 2, 3 and 4. In other words, the terms of the form $\sim \lambda_{i} \lambda_{j}$ do not seem to play a fundamental role in the flow equation at the scales of $k$ reported here. Neglecting the contributions from the $\rho$ and pomeron channels does therefore not seem to be a too brutal approximation.

We have also tried to test the approximation involved in using the two-loop expression for the running coupling constant. In order to do this, we have considered a different version of the running coupling constant in the flow equation (2.31): we have taken for 
$g_{k}^{2}\left(p^{2}\right)$ the usual two-loop expression when $\left.g_{k}^{2}\left(p^{2}\right)\right|_{2-l o o p}<10$, while $g_{k}^{2}\left(p^{2}\right)=10$ when $\left.g_{k}^{2}\left(p^{2}\right)\right|_{2-\text { loop }}>10$. In other words, the new $g_{k}^{2}\left(p^{2}\right)$ stops running when it reaches the value $g_{k}^{2}=10$. Also in this case, the solution $\lambda_{\sigma}(t)$ of the modified flow equation does not show any dramatic difference when compared to the solution of Eq. (2.31) reported in Figs. 2, 3 and 4.

Finally, since the one-particle irreducible four-point function remains small in the momentum range of interest, a neglection of the higher-order anomaly-free effective multiquark vertices seems well motivated.

\section{Comparison with the instanton contribution}

In Eq. (2.25) we have chosen to parametrize $\Gamma_{4, k}^{(F)}[\psi, \bar{\psi}]$ by using $U\left(N_{f}\right) \otimes U\left(N_{f}\right)$ chirally symmetric operators $\mathcal{M}_{\sigma}, \mathcal{M}_{\rho}$ and $\mathcal{M}_{p}$. In this section we supplement the effect of the chiral anomaly. We add to the effective four-quark interactions an anomaly-induced term [15, which is $S U(2) \otimes S U(2) \otimes U(1)_{V}$ invariant, but not $U(1)_{A}$ invariant.

In particular, for a nonvanishing mass $m_{s}$ of the strange quark, the instanton-induced fermion effective action for $N_{f}=3$ includes a four-fermion contribution of the form [16]

$$
\Gamma_{\text {inst }}^{(4)}=\int \mathrm{d}^{4} x \lambda_{\text {inst }}^{\left(N_{c}\right)} \mathcal{O}_{4, \text { inst }}^{\left(N_{c}\right)}(x)
$$

where

$$
\begin{aligned}
& \mathcal{O}_{4, \text { inst }}^{\left(N_{c}\right)}=\left(\delta_{i}^{j} \delta_{k}^{l}-\frac{1}{N_{c}} \delta_{i}^{l} \delta_{k}^{j}\right) \times \\
& \quad \times\left\{\left[\bar{u}^{i} u_{j}\right]\left[\bar{d}^{k} d_{l}\right]-\left[\bar{u}^{i} d_{j}\right]\left[\bar{d}^{k} u_{l}\right]+\left[\bar{u}^{i} \gamma^{5} u_{j}\right]\left[\bar{d}^{k} \gamma^{5} d_{l}\right]-\left[\bar{u}^{i} \gamma^{5} d_{j}\right]\left[\bar{d}^{k} \gamma^{5} u_{l}\right]\right\},
\end{aligned}
$$

This operator comes from $\Delta L_{3, I}^{\left(N_{c}\right)}+\Delta L_{3, A}^{\left(N_{c}\right)}$, where $I=$ instanton and $A=$ anti-instanton, and the effective coupling constant involves an integral over instanton sizes $\rho$ :

$$
\begin{aligned}
\lambda_{\text {inst }}^{\left(N_{c}\right)} & =\frac{(1.34)^{3} m_{s}}{2\left(N_{c}^{2}-1\right)} \int_{0}^{1 / k} \frac{\mathrm{d} \rho}{\rho^{4}} d_{0}^{\left(N_{c}\right)}(\rho)\left(4 \pi^{2} \rho^{3}\right)^{2}\left(\frac{\alpha(1 / \rho)}{\alpha(\bar{\mu})}\right)^{-\frac{1}{4 \pi^{2} \beta_{0}}} \\
& =0.35 \cdot \frac{m_{s}}{k^{3}} \int_{0}^{1} \mathrm{~d} z z^{2}\left(\frac{\alpha(k / z)}{\alpha(\bar{\mu})}\right)^{-\frac{4}{9}}\left[\frac{2 \pi}{\alpha(k / z)}\right]^{6} \exp \left[-\frac{2 \pi}{\alpha(k / z)}\right] .
\end{aligned}
$$


Here $\bar{\mu}=1 \mathrm{GeV}$ is the renormalization scale for the fermion fields and [16, 17]:

$$
d_{0}^{\left(N_{c}\right)}(\rho)=C_{N_{c}}\left[\frac{2 \pi}{\alpha(1 / \rho)}\right]^{2 N_{c}} \exp \left[-\frac{2 \pi}{\alpha(1 / \rho)}\right]
$$

with

$$
C_{N_{c}}=\frac{4.6 \exp \left(-1.68 N_{c}\right)}{\pi^{2}\left(N_{c}-1\right) !\left(N_{c}-2\right) !} .
$$

For $\alpha(1 / \rho)$ we take the (two-loop) running coupling constant (2.34) with $\mu=1 / \rho$. For the mass of the strange quark we have used the value $m_{s} \simeq 150 \mathrm{MeV}$. For $N_{c}=3$ and $N_{f}=3$ one has $C_{3} \simeq 1.51 \times 10^{-3}$ and we use (2.37). We have integrated the instanton length scale $\rho$ between 0 and $1 / k$, since $k$ is an infrared cutoff momentum scale. The long-dashed line in Fig. 2 is the value of $\lambda_{\text {inst }}^{(3)}$ (which is $t$-independent!) as a function of $k$. One concludes that the instanton-induced four-quark interaction becomes dominant at a scale $k$ around (somewhat below) $800 \mathrm{MeV}$.

\section{Spontaneous color symmetry breaking?}

It has been suggested [10] that confinement can be described by the Higgs phenomenon. In this picture the expectation value of a color-octet quark-antiquark condensate $\chi_{0}$ gives a mass to the gluons

$$
M^{2}=g_{e f f}^{2} \chi_{0}^{2}
$$

In the limit of three massless quarks the mass of all eight gluons is equal. Due to spontaneous color symmetry breaking the physical electric charge of the gluons is integer and they carry precisely the quantum numbers of the known vector mesons $\mathrm{f}^{\Uparrow} \rho, K^{*}, \omega$. For this reason $M$ is identified with the average mass of the vector meson octet $M_{V} \approx 850 \mathrm{MeV}$. Confinement arises in this picture in complete analogy to magnetic flux tubes in superconductors. A nonlinear reformulation of the Higgs phenomenon preserves manifestly the gauge symmetry and shows the equivalence of the confinement and Higgs pictures [18]. Instanton-induced six-quark interactions destabilize the "color symmetric vacuum" and have been proposed as the origin of dynamical color symmetry breaking.

\footnotetext{
${ }^{*}$ See [10] for a discussion of the singlet vector meson state.
} 
The results in the preceeding sections correspond to a vanishing octet condensate $\chi_{0}=0$. In order to get some information on the $\chi_{0}$-dependence of the effective four-quark interactions we present in this section results for a nonvanishing value of the induced gluon mass term $M_{k}^{2}$. Since the precise relation between $g_{\text {eff }}$ in Eq. (5.1) and the running gauge coupling in our approximation (2.37) is nontrivial [10, we present results for two different settings.

For the first setting (A) we simply add a constant gluon mass term $M_{k}^{2}=M_{V}^{2}=$ $(850 \mathrm{MeV})^{2}$ in the propagator such that:

$$
m_{k}^{2}=M_{V}^{2}+m_{k A}^{2}+m_{k F}^{2}
$$

Moreover, we use a momentum-dependent running gauge coupling $\alpha(\mu)$, with $\mu^{2}=q^{2}+$ $k^{2}+M_{V}^{2}$. For the second setting (B) we work at constant $\chi_{0}=140 \mathrm{MeV}$ with a running perturbative gauge coupling (2.34) $g_{k}^{2}=4 \pi \alpha$, i.e., $M_{k}^{2}=g_{k}^{2} \chi_{0}^{2}$. This replaces the gluon mass term in Eq. (2.20) by

$$
m_{k}^{2}=g_{k}^{2} \chi_{0}^{2}+m_{k A}^{2}+m_{k F}^{2}
$$

In this case, we use a momentum-dependent running gauge coupling $\alpha(\mu)$, with $\mu^{2}=$ $q^{2}+k^{2}+\bar{M}_{k}^{2}$, where $\bar{M}_{k}^{2}=4 \pi \alpha(\bar{\mu}=600 \mathrm{MeV}) \cdot \chi_{0}^{2}$.

In Figs. 5 and 6 we compare $\lambda_{\sigma}$ and $\lambda_{\sigma}^{(1 g)}$ for different running gluon mass terms $m_{k}^{2}$ given by (5.2) (A), (5.3) (B), and (2.20) (C). We observe that the gluon mass damps the increase of both $\lambda_{\sigma}$ and $\lambda_{\sigma}^{(1 g)}$. On the other hand, a pointlike approximation to the anomaly-free four-quark interaction becomes now better justified, especially for (A). Thanks to the additional infrared cutoff $M_{k}^{2}$ the flow can formally be followed towards $k \rightarrow 0$ without encountering a problem from a diverging gauge coupling: the growth of the gauge coupling is reduced by the presence of the (nonvanishing) gluon mass term. One should not expect, however, that our results remain valid for very small $k$. Indeed, it has been argued that the quark wave function renormalization $Z_{\psi, k}$ multiplying the quark kinetic term门 (2.24) drops by a factor of about three as $k$ reaches a few hundred $\mathrm{MeV}$. This reflects the binding of three quarks into a baryon. As a result, the running gauge coupling $g_{k}$ increases by a factor of three and the renormalized four-quark couplings $\lambda_{\sigma}$ and $\lambda_{\sigma}^{(1 g)}$ become multiplied by a factor of nine. The running of the wave function

\footnotetext{
†This wave function has been set $Z_{\psi, k}=1$ for the present work.
} 
renormalization becomes therefore important for low $k$. We have not included it here (except for its contribution to the running gauge coupling), and this is the reason why we do not explore very low values of $k$ even though the infrared problems are formally absent in presence of a nonzero gluon mass.

We finally note that, in presence of six quark interactions reflecting the axial anomaly, an octet condensate $\chi_{0} \neq 0$ will also result in additional effective four-quark interactions linked to the anomaly. They are not included in the present investigation. We estimate their order of magnitude to be comparable to (or even larger than) the instanton contribution discussed in Sect. 4.

\section{Summary and conclusions}

In this paper we have explored the effective Nambu-Jona-Lasinio-type four-quark interactions at the nonperturbative scale $\Lambda_{\mathrm{NJL}} \approx(500-800) \mathrm{MeV}$. For this purpose we have used a method based on truncations of the exact nonperturbative renormalization group equation for the effective average action 4,6 , 8]. More precisely, we have derived a truncated nonperturbative flow equation for the scalar-like effective momentum-dependent four-quark interaction in QCD. This type of interaction is relevant for the physics of scalar and pseudoscalar mesons and therefore for spontaneous chiral symmetry breaking.

The nonperturbative renormalization group equation describes how the momentum dependent four-quark vertex depends on an infrared cutoff $k$. The initial value of the vertex at short distances is computed from perturbative QCD. We have studied the abovementioned flow equation both analytically, in the perturbative regime, and numerically, in the nonperturbative regime. For the nonperturbative regime we have derived the dependence of the scalar-like four-quark interaction on the infrared cutoff scale $k$ and on the momentum exhanged in the $t$-channel. The results are summarized in Figs. 2, 3 and 4.

We find that a quasilocal one-particle-irreducible (1PI) four-quark interaction is generated and becomes indeed approximately pointlike for scales of the order $\Lambda_{\mathrm{NJ}}$, where scalar and pseudoscalar meson-bound states are expected to play a role. The "quasilocality" of this "box-interaction" is put in evidence in Fig. 4, where the dependence on the 
momentum-scale is shown.

As discussed in Sect. 4, another pointlike interaction arises from instanton effects. In Fig. 2 a comparison is made between the strength of the box interaction and that of the local instanton-induced four-quark interaction. At scales of the order $\Lambda_{\mathrm{NJL}}$, the instanton interaction dominates.

Nevertheless, the contribution of the one-gluon exchange to the effective four-quark vertex retains the characteristic momentum dependence of a particle exchange in the $t$ channel. We show the different contributions to the four-quark vertex in Figs. 2, 3 and 4. The effective one-gluon exchange contribution remains substantially larger than the pointlike box-type interactions. In consequence, the pointlike interactions (1.1) of the Nambu-Jona-Lasinio model cannot give a very accurate description of QCD if the gluons are massless. Models based on a pointlike instanton induced four-quark interaction look more favorable. Still, the one-gluon exchange is not negligible.

The results shown in Figs. 2, 3 and 4 have been obtained with the assumption of massless gluons (in the limit of vanishing infrared cutoff scale, $k \rightarrow 0$ ). In Sect. 5 we have discussed the intriguing hypothesis that gluons acquire a mass $M_{V} \approx 850 \mathrm{MeV}$ from spontaneous color symmetry breaking [10]. Our results for a nonvanishing gluon mass are shown in Figs. 5 and 6. In particular, from Fig. 6, where the dependence on the momentum scale is shown, it is clear that for this scenario pointlike four-quark interactions can be defended as a much better approximation. Nevertheless, any quantitatively reliable approximation should retain the one-gluon exchange in addition to the instanton interaction and the NJL-interaction (1.1).

We emphasize that our approximations for the flow equation in the nonperturbative range remain relatively crude, even if in Sect. 3 we have done some checks in order to test the validity of some of the many approximations involved. Our results should therefore only be trusted on a semi-quantitative level. In our opinion, they are nevertheless a good guide for the qualitative characteristics of the effective four-quark interactions at scales between a few hundred $\mathrm{MeV}$ and $1 \mathrm{GeV}$. 


\section{References}

[1] Y. Nambu and G. Jona-Lasinio, Phys. Rev. 122 (1961) 345.

[2] T. Hatsuda and T. Kunihiro, Phys. Rep. 247 (1994) 221;

J. Bijnens, Phys. Rep. 265 (1996) 369.

[3] J. Berges, D. Jungnickel and C. Wetterich, Phys. Rev. D 59 (1999) 034010.

[4] C. Wetterich, Phys. Lett. B 301 (1993) 90; Nucl. Phys. B 352 (1991) 529; Z. Phys. C 57 (1993) 451; C 60 (1993) 461.

[5] J. Berges, N. Tetradis and C. Wetterich, hep-ph/0005122.

[6] M. Reuter and C. Wetterich, Nucl. Phys. B 408 (1993) 91; B 417 (1994) 181.

[7] M. Bonini, M. D’Attanasio and G. Marchesini, Nucl. Phys. B 418 (1994) 81.

[8] U. Ellwanger, Phys. Lett. B 335 (1994) 364.

[9] U. Ellwanger and C. Wetterich, Nucl. Phys. B 423 (1994) 137.

[10] C. Wetterich, Phys. Lett. B 462 (1999) 164; hep-ph/9908514 (to appear in Eur. Phys. J. C); hep-ph/0008150; hep-ph/0011076.

[11] C. Wetterich, hep-th/9501119.

[12] C. Wetterich, Z. Phys. C 72 (1996) 139.

[13] U. Ellwanger, M. Hirsch and A. Weber, Z. Phys. C 69 (1996) 687.

[14] B. Bergerhoff and C. Wetterich, Phys. Rev. D 57 (1998) 1591.

[15] G. 't Hooft, Phys. Rev. Lett. 37 (1976) 8;

G. 't Hooft, Phys. Rev. D 14 (1976) 3432; D 18 (1978) 2199 (Erratum).

[16] M.A. Shifman, A.I. Vainshtein and V.I. Zakharov, Nucl. Phys. B 163 (1980) 46.

[17] E.V. Shuryak, Nucl. Phys. B 203 (1982) 93. 
[18] T. Banks and E. Rabinovici, Nucl. Phys. B 160 (1979) 349;

E. Fradkin and S. Shenker, Phys. Rev. D 19 (1979) 3682;

G. 't Hooft, in: Recent Developments in Gauge Theories (Plenum, New York, 1980), p. 135 ;

S. Dimopoulos, S. Raby and L. Susskind, Nucl. Phys. B 173 (1980) 208;

T. Matsumoto, Phys. Lett. 97B (1980) 131;

M. Yasuè, Phys. Rev. D 42 (1990) 3169. 


\section{FIGURE CAPTIONS}

Fig. 1. One-loop diagrams for the flow equation (2.31) for the four-fermion (1PI) coupling $\lambda_{\sigma}$. Five more diagrams similar to c) with gluon lines attached at different quark lines are not shown.

Fig. 2. Scale dependence of the quartic four-quark coupling.

Continuous line: $\lambda_{\sigma}(t=0)$ as a function of $k$ with the initial condition as in Eqs. (3.1), (3.7). Dotted line: $\lambda_{\sigma}(t=0)$ as a function of $k$ with the initial condition $\left.\lambda_{\sigma}(t)\right|_{k=k_{c}}=0[\operatorname{ansatz}(\mathrm{A})]$. Dot-dashed line: the same as for the continuous line, but using everywhere (both in the flow equation and in the initial condition) the momentum-independent coupling constant $\alpha[\operatorname{ansatz}(\mathrm{B})]$. Dashed line: the onegluon-exchange contribution $\lambda_{\sigma}^{(1 g)}(t=0)$, defined by Eq. (2.41), as a function of

k. Long-dashed line: the instanton contribution $\lambda_{\text {inst }}^{(3)}$, defined by Eq. (4.3), as a function of $k$.

Fig. 3. Different approximations for $\lambda_{\sigma}$ in perturbation theory.

Continuous line: the same as in Fig. 2. Dotted line: $\lambda_{\sigma}^{(p)}(t=0)$ versus $k$, momentumdependent $\alpha[\operatorname{ansatz}(\mathrm{A})]$. Dashed line: $\lambda_{\sigma}^{(p)}(t=0)$ versus $k$, momentum-independent $\alpha[\operatorname{ansatz}(\mathrm{B})]$. Dot-dashed line: $\lambda_{\sigma}^{(p)}(t=0)$ versus $k$, constant $\alpha[\operatorname{ansatz}(\mathrm{C})]$.

Fig. 4. Dependence of the four-quark interaction on the transferred momentum $t$.

We show $\lambda_{\sigma}(t)$ (continuous line) and the one-gluon-exchange contribution $\lambda_{\sigma}^{(1 g)}(t)$ (dashed line) as functions of $t$ at the fixed value $k=0.42 \cdot \Lambda_{Q C D} / \Lambda \frac{(3)}{\mathrm{MS}} \mathrm{GeV}$.

Fig. 5. Quartic coupling with spontaneous color symmetry breaking.

Continuous and dashed lines: the same as in Fig. 2. Lower (A) and upper (B) dotted lines: $\lambda_{\sigma}(t=0)$ versus $k$ using the running gluon mass term $m_{k}^{2}$ given by Eq. (5.2) (A) and Eq. (5.3) (B). Lower (A) and upper (B) dot-dashed lines: $\lambda_{\sigma}^{(1 g)}(t=0)$ versus $k$ using the running gluon mass term $m_{k}^{2}$ given by Eq. (5.2) (A) and Eq. (5.3) (B). 
Fig. 6. Momentum dependence of quartic couplings with spontaneous color breaking. Continuous and dashed lines: the same as in Fig. 4. Lower (A) and upper (B) dotted lines: $\lambda_{\sigma}(t)$ versus $t$ using the running gluon mass term $m_{k}^{2}$ given by Eq. (5.2) (A) and Eq. (5.3) (B). Lower (A) and upper (B) dot-dashed lines: $\lambda_{\sigma}^{(1 g)}(t)$ versus $t$ using the running gluon mass term $m_{k}^{2}$ given by Eq. (5.2) (A) and Eq. (5.3) (B). 
Figure 1
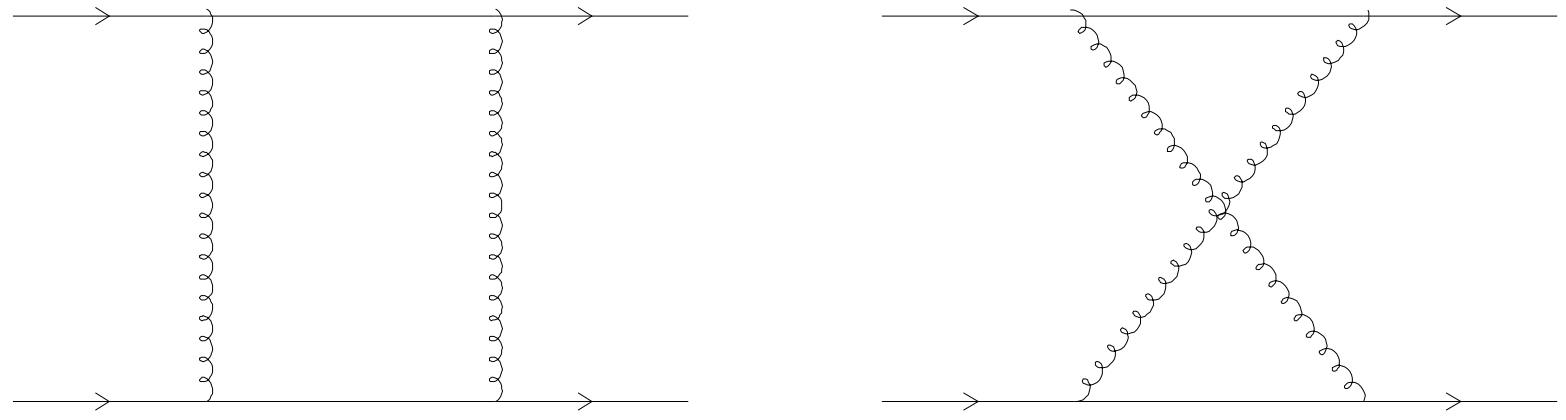

a)

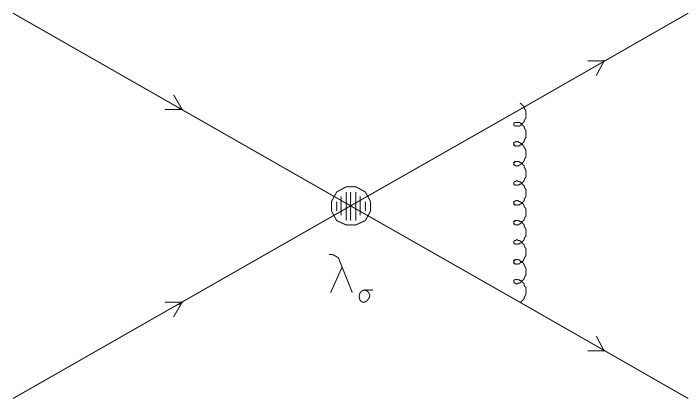

b)

c)

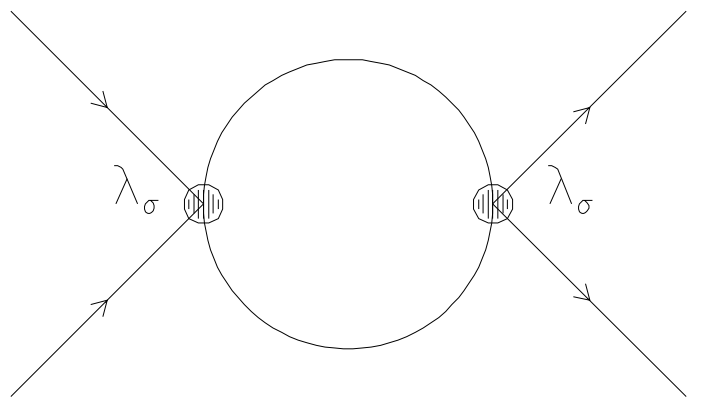

d) 


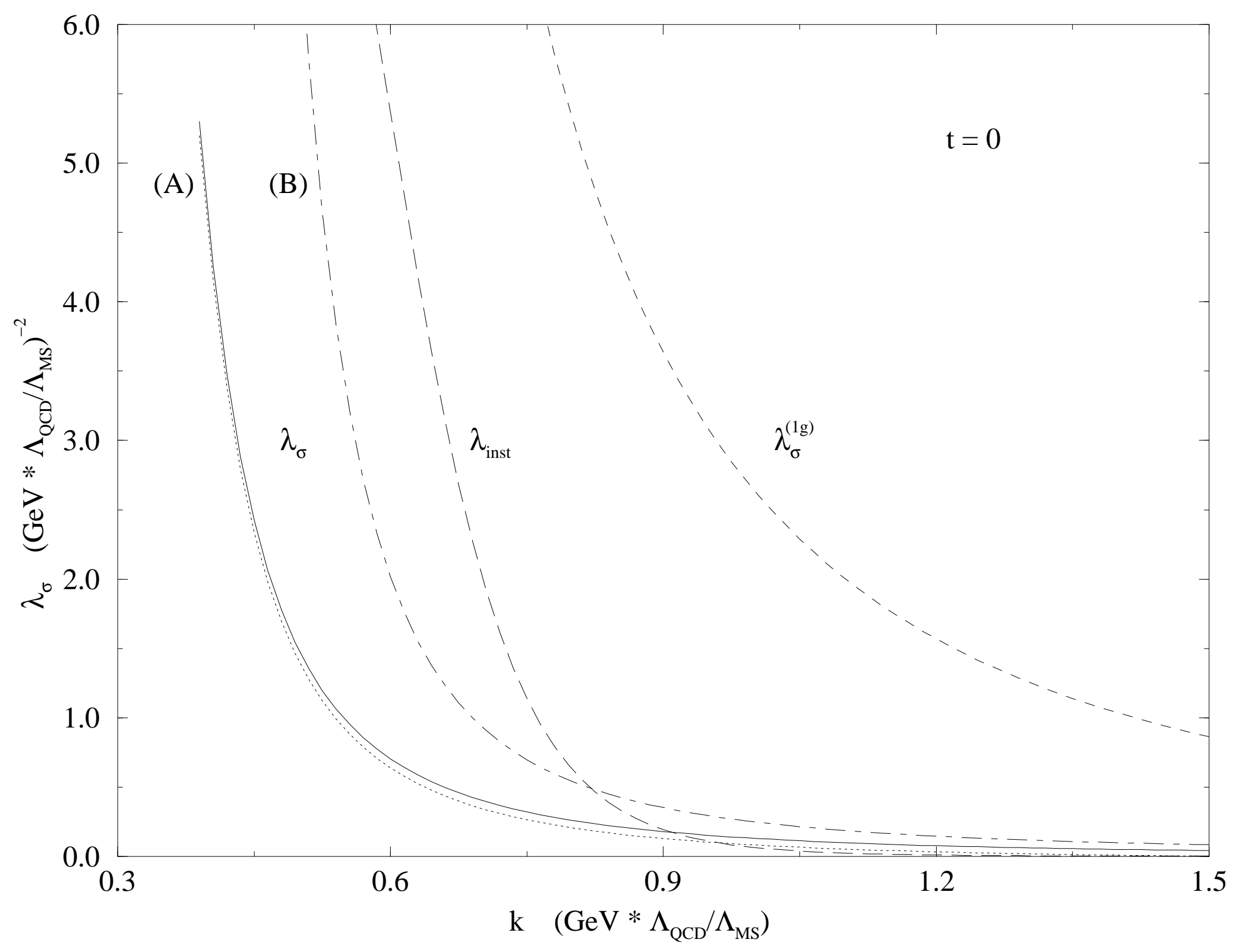

0.7
0
0
0
0
0
0 


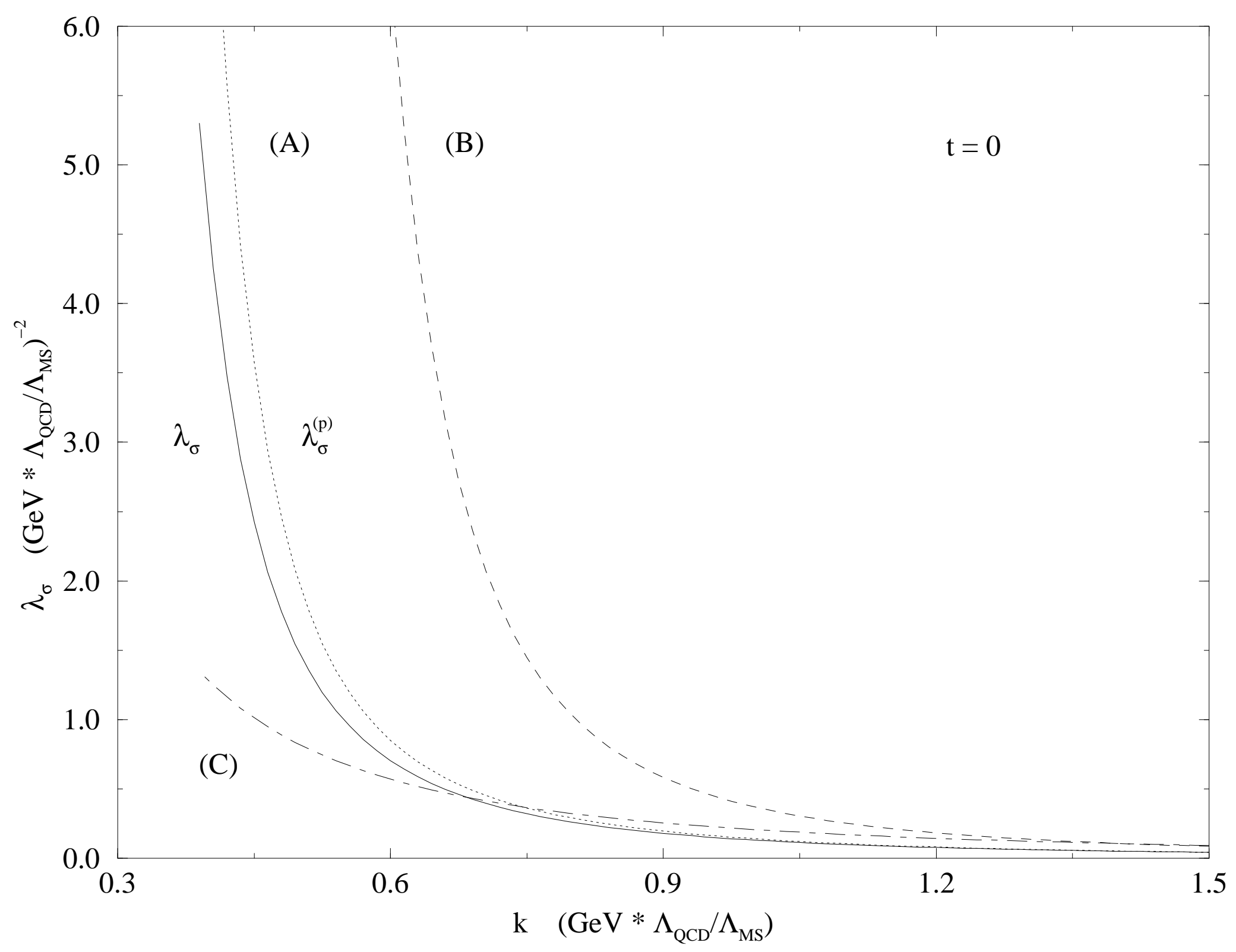

ب0. 


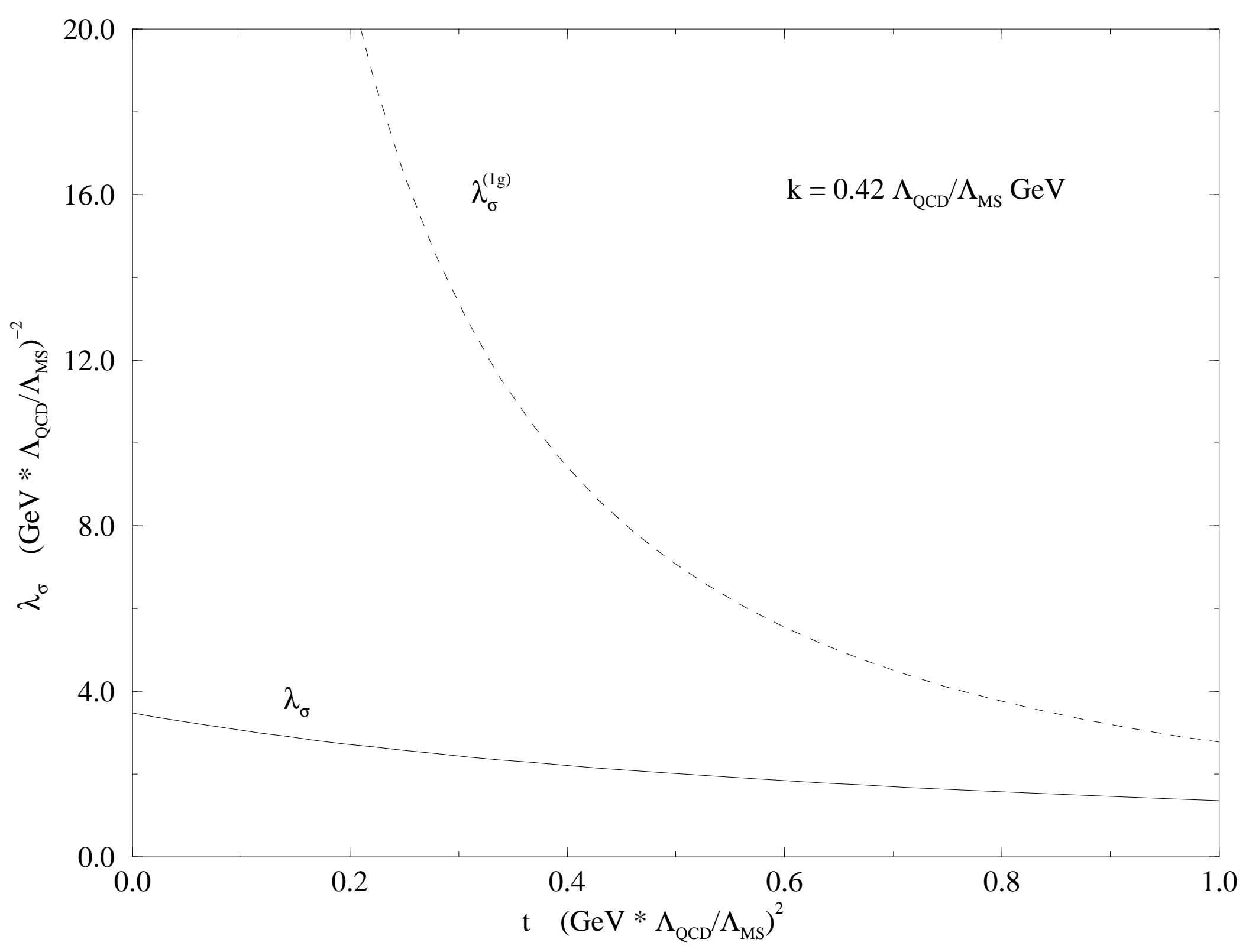

2
0
0
0
0
0
0
0 


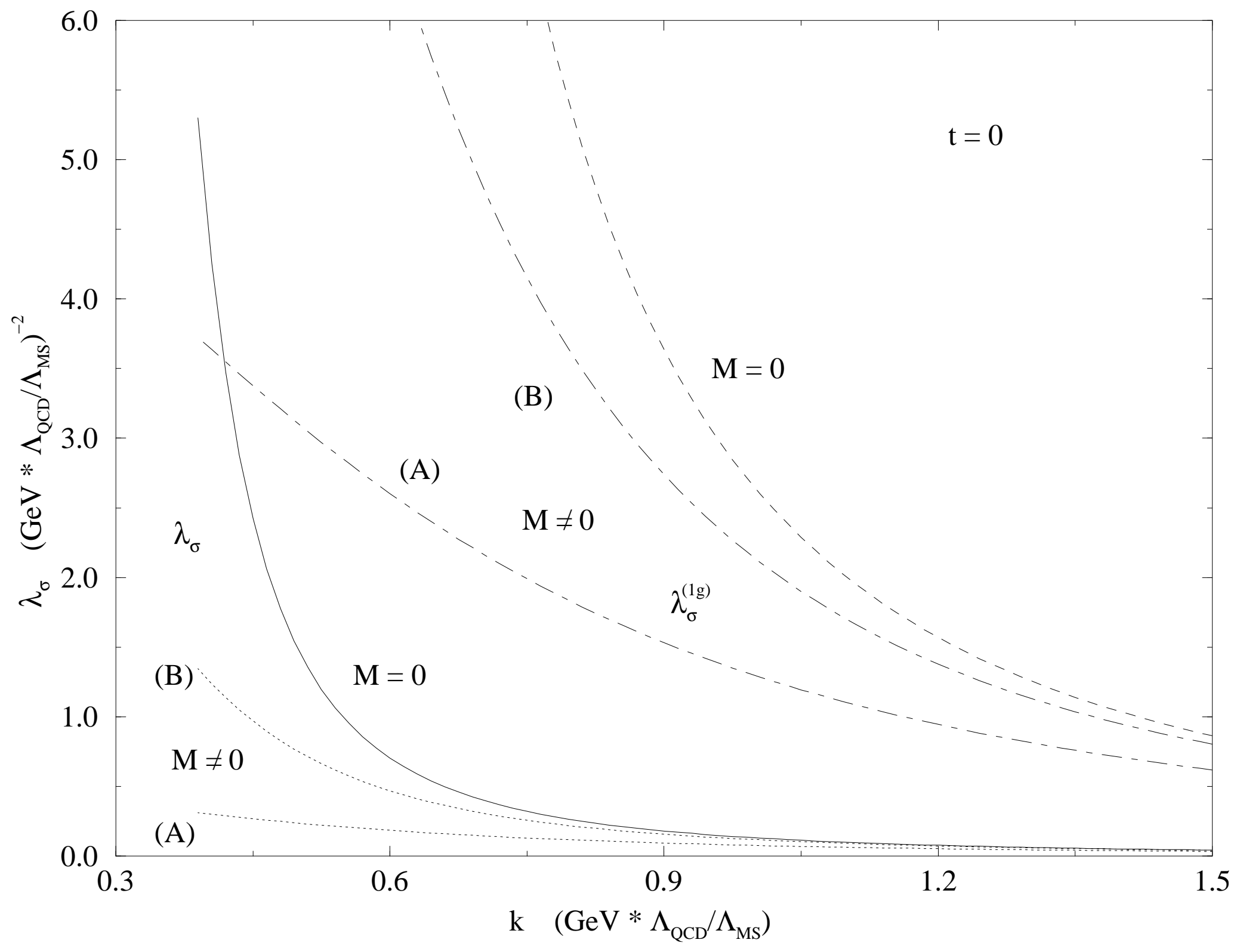

0
0
0
0
0 


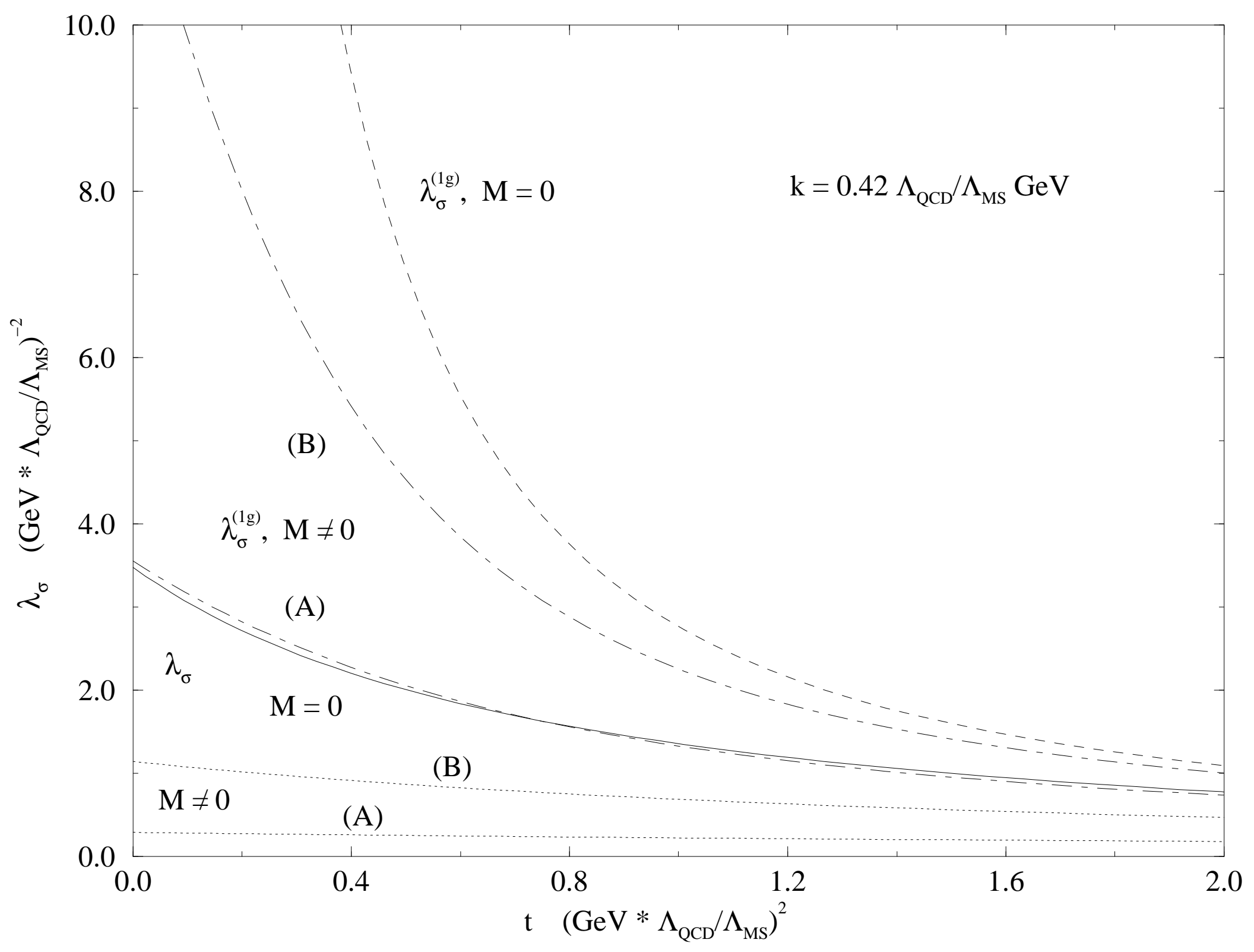

0.0
0
0
0
0
0 